\title{
FIRST EXPERIENCES WITH ACTIVE C-BAND RADAR REFLECTORS AND SENTINEL-1
}

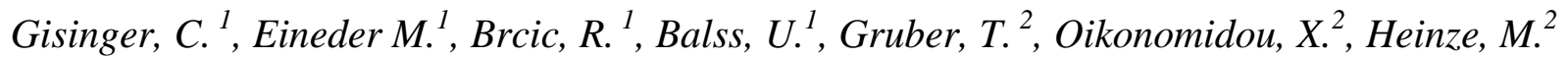 \\ ${ }^{1}$ Remote Sensing Technology Institute, German Aerospace Center (DLR) \\ ${ }^{2}$ Chair of Astronomical and Physical Geodesy, Technical University of Munich (TUM)
}

\begin{abstract}
We report our first results with Sentinel-1 Interferometric Wide Swath (IW) data using novel off-the-shelf electronic corner reflectors (ECRs) for geometric measurements with C-band Synthetic Aperture Radar (SAR). At the German Aerospace Center (DLR) campus in Oberpfaffenhofen, we set up an arrangement consisting of two trihedral corner reflector and two active ECRs. We describe the practical aspects of such ECRs as well as first radiometric characteristics. Moreover, we present geometric accuracy as derived from imaging geodesy, i.e. absolute radargrammetric positioning in $2 \mathrm{D}$ and $3 \mathrm{D}$, as well as interferometric phase measurements.
\end{abstract}

Index Terms-Active Radar Transponder, Electronic Corner Reflector, Absolute Ranging, Imaging Geodesy, Synthetic Aperture Radar, SAR, Geodetic Height System

\section{INTRODUCTION}

Corner reflectors (CRs) are well established passive devices, commonly used for the radiometric and geometric calibration of SAR. Their radar cross section is large, wellknown and rather independent of the incidence angle. In addition, the phase center is exactly at the intersection of the orthogonal planes. Once robustly mounted, they provide a stable reference over years. However, there are also disadvantages: Due to their mechanical size and weight, CRs are sometimes inconvenient or impossible to install, e.g. on steep unstable mountain slopes or near buildings in urban or industrial environments. In such environments, smaller devices are desirable and due to their smaller antenna size, active radar transponders are an alternative. Furthermore, observations from ascending and descending pass geometries require two $\mathrm{CRs}$, whereas a single ECR can support both directions.

In the frame of the ESA project SAR-HSU (ESA AO/19172/17/I-BG-Baltic+) with the goal of monitoring tide gauges with SAR to connect the height systems of neighboring states (Sweden, Finland, Poland, Estonia,) some are severely influenced by postglacial uplift - a test installation with 10 ECRs surrounding the Baltic Sea is planned. Due to the long baselines and large waterbody between them, SAR interferometry cannot be used for differential height change measurements. Instead absolute SAR measurements for each single point are evaluated. The technique has been demonstrated to achieve cm-level accuracy with high resolution TerraSAR-X data [1], [2] and a ranging accuracy of about $6 \mathrm{~cm}$ with Sentinel-1 data [3]. While we have demonstrated this accuracy with CRs, no experience exists so far with active ECRs.

In this paper we describe our first experiences with setting up a test field of ECRs at the DLR campus in Oberpfaffenhofen and report on operational experiences and first quantitative measurement results.

\section{ECRS FROM METASENSING}

In 2018 we purchased four C-band ECRs from MetaSensing. The devices measure about $60 \mathrm{~cm}$ x $40 \mathrm{~cm}$ x $30 \mathrm{~cm}$ and can handle ascending and descending passes in horizontal $(\mathrm{HH})$ or vertical (VV) co-polarized operations. Their Radar Cross Section (RCS) in C-band is on the order of $42 \mathrm{dBm}^{2}$, comparable to a passive trihedral $\mathrm{CR}$ with an inner leg dimension of $1.8 \mathrm{~m}$ [4]. The switch-on times and modes of operation are pre-programmed according to SAR satellite passes and are controlled by a microcontroller which is powered by built-in rechargeable batteries. The battery can be recharged either by a solar panel or by a permanent $230 \mathrm{~V}$ line connection. Command and control is performed either via a USB interface or WiFi connection.

\section{ON-SITE INSTALLATION}

The operation of active radar devices in Europe requires a dedicated license. For our experiments in Germany, we received a temporary license from the responsible German authority (Bundesnetzagentur). We are also pursuing an unlimited license for Germany.

Our set-up consists of two 1.5 m CR and two ECRs located at the DLR Oberpfaffenhofen campus, with baselines of approximately 200 to 900 meters, see Fig.1. Since we desire to achieve accuracy on the order of centimeters in absolute ranging mode and even millimeters in spatial relative interferometric mode, a stable mounting is required. 


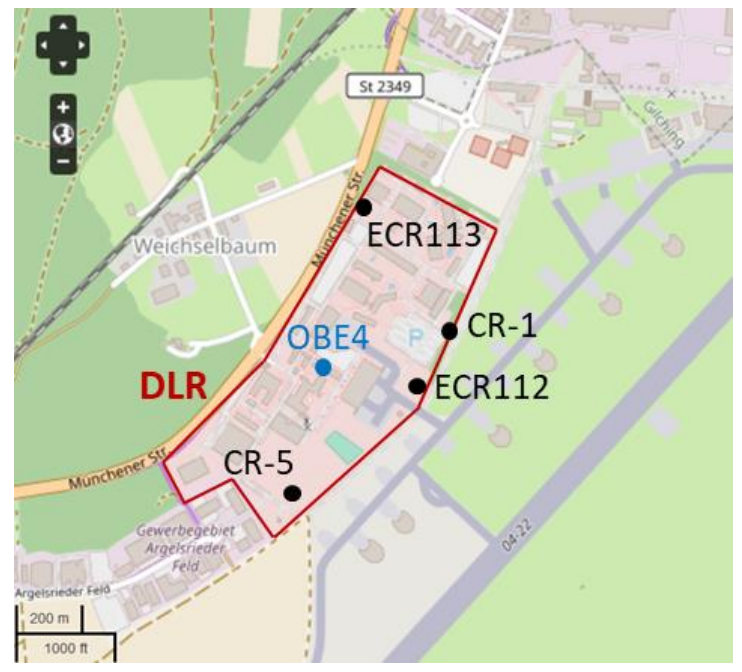

Fig. 1: ECR and CR installations at DLR Oberpfaffenhofen.

While the CRs could only be anchored in soil without concrete foundation, the ECRs are mounted on metal pillars of $130 \mathrm{~m}$ length, which are drilled into the ground to a depth of about $80 \mathrm{~cm}$. On top of the pillar is a level platform where the ECR is mounted, see Fig.2. From there it can be removed for inspection and replaced with an accuracy of $<1$ $\mathrm{mm}$. The ECR platforms are accurately aligned to geographic North $\left(<2^{\circ}\right)$ whereas the CRs are oriented to the zero-Doppler plane to support ascending pass geometries of Sentinel-1. Periodic command and control (inspection of the ECR web interface and extraction of logfiles) are performed on-site via WiFi to monitor the status of the devices.

The position of both ECRs and CR-1 are known from DGPS survey. We measured the pillar central positions at the height of the ECR base plate as well as the phase center position of the CR by mounting a geodetic GPS for 2-3 hours at each location. Processing was performed with respect to the permanent station OBE4, also contributing to the International GNSS Service (IGS). The precision as reported by the Leica GNSS software we used to derive the coordinates is better than $2 \mathrm{~cm}$.

\section{FIRST OPERATIONAL EXPERIENCES}

\subsection{Power Supply}

During early laboratory experiments, the internal battery was unfortunately found to be unsuitable for supporting operations longer than several weeks. With nominal Sentinel-1 acquisitions executed every couple of days we expect power support for approximately 2-3 weeks. In order to finish this experiment earlier we programmed hourly activations of 10 minute duration each after day 4 . On day 5 after 35 activations the capacity dropped to $30 \%$ and the device failed and rebooted. Hence, in this experiment we achieved about 350 minutes of active ECR operation time.

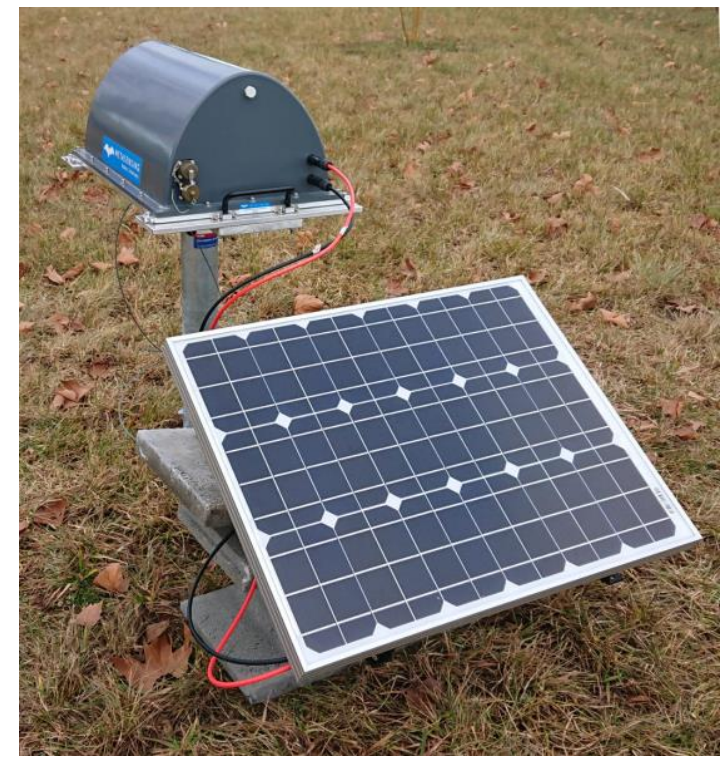

Fig. 2: ECR112 installation with solar panel power supply.

Careful planning and minimization of activation times could certainly provide a longer standby time based purely on battery power, but from our experience, even without activations the battery would deplete after a couple of days.

In consequence we fitted our ECRs with external power supplies, ECR113 with a permanent line connection, ECR112 with a 50W solar panel. The solar power supply provided sufficient energy over weeks and never failed. However in continuous low light conditions the device might run out of power. We assume that it would recover as soon as power becomes available again, but so far we did not test this. With the external power supplies installed, ECR113 managed faultless continuous operation and is still operating as of today, whereas ECR112 stopped working after 1.5 months on February $25^{\text {th }}$ and had to be sent back to MetaSensing for repair. The cause of failure was identified and the device will be reinstalled in June to continue parallel ECR operation.

\subsection{Control Data handling}

Due to initial power problems und some spurious reboots with early firmware versions we decided to monitor the devices closely using the WiFi interface. With the most recent firmware v2.15, internal buffers of $2 \times 250 \mathrm{kB}$ store all logging information reported by the device in ASCII format, among them the activation times, GPS-based system time updates, hourly battery status, temperature and humidity. After about 40 days of undisturbed routine operations, the active log fills up the available space and is moved to a backup buffer, which can still be retrieved. In order to preserve all information, the backup buffer should be retrieved every 4-5 weeks, or both buffers after 8 weeks, maximum. 


\section{RADAR CROSS SECTION}

For the selection of CRs and ECRs, the Signal to Clutter Ratio (SCR) has to be determined and for Sentinel-1 it should be larger than $25 \mathrm{~dB}$ in order to achieve an absolute ranging error of $6 \mathrm{~cm}$ [4]. Indeed the RCS of the ECRs is large enough to easily recognize them in sub-urban environments. Only large industrial facades oriented towards the SAR generate comparably bright signatures. In numbers, ECR-113 was determined to be $5.4 \mathrm{~dB}$ brighter than CR-1 with a measured RCS of $37.0 \mathrm{dBm}^{2}$.

\section{ABSOLUTE 2D GEOLOCATION RESULTS}

The geolocation quality of SAR sensors can be evaluated by comparing the reference coordinates of point targets with the measured image data in 2D SAR image space (range and azimuth). Our analysis is performed using the precise orbit solution of Sentinel-1 as well as corrections for atmospheric path delays, solid Earth tide deformations, and sensor specific system corrections. The details of our methods are summarized in [3], [5].

Over Europe Sentinel-1A/B acquires data continuously with overlapping swaths. At the DLR test site this allows us to analyze data from two ascending geometries $\left(31^{\circ} \& 40^{\circ}\right)$ and one descending geometry $\left(38^{\circ}\right)$, each with a temporal sampling of 6 days. For the two ECRs and CR-1, for which the coordinates are known from the survey, geolocation results are presented in Table 1 and Fig. 3 for a total of 63 single look complex (SLC) IW image products. The products cover a period of four months, i.e. January 2020 to mid-May 2020. ECR112 was only analyzed for 20 images, due to its failure.

Notably, the ECRs show smaller standard deviations in azimuth, which suggests a benefit from their larger RCS. On the other hand, they perform worse in range than CR-1. The range standard deviations of the ECRs for the individual stacks are 2-3 times larger than the $0.034 \mathrm{~m}$ achieved with CR-1. The outcomes for CR-1 are in-line with our previous findings [3]. Moreover, we observe meter level range offsets for the ECRs, whereas CR-1 agrees very well with the surveyed coordinates, see Fig.3.

Table 1: 2D geolocation results for ECR112, ECR 113 and CR1 using Sentinel-1 IW SLC data; mean value \pm standard deviation per stack and across all data.

\begin{tabular}{rrrrrrr}
\hline Stack & \multicolumn{3}{c}{ Range [m] } & \multicolumn{3}{c}{ Azimuth [m] } \\
& $\mathrm{ECR}_{112}$ & $\mathrm{ECR}_{113}$ & \multicolumn{1}{c}{$\mathrm{CR}_{1}$} & $\mathrm{ECR}_{112}$ & $\mathrm{ECR}_{113}$ & $\mathrm{CR}_{1}$ \\
\hline $\mathrm{A} 31^{\circ}$ & 2.138 & 1.783 & -0.012 & 0.092 & 0.409 & 0.486 \\
& \pm 0.118 & \pm 0.070 & \pm 0.033 & \pm 0.124 & \pm 0.298 & \pm 0.374 \\
$\mathrm{~A} 40^{\circ}$ & 2.178 & 1.896 & -0.029 & 0.149 & 0.100 & -0.005 \\
& \pm 0.148 & \pm 0.075 & \pm 0.034 & \pm 0.168 & \pm 0.168 & \pm 0.437 \\
$\mathrm{D} 38^{\circ}$ & 1.472 & 1.234 & & 0.309 & -0.192 & \\
& \pm 0.131 & \pm 0.115 & & \pm 0.153 & \pm 0.253 & \\
\hline Total & 1.881 & 1.636 & -0.020 & 0.193 & 0.110 & 0.246 \\
& \pm 0.365 & \pm 0.303 & \pm 0.034 & \pm 0.171 & \pm 0.350 & \pm 0.472 \\
\hline
\end{tabular}

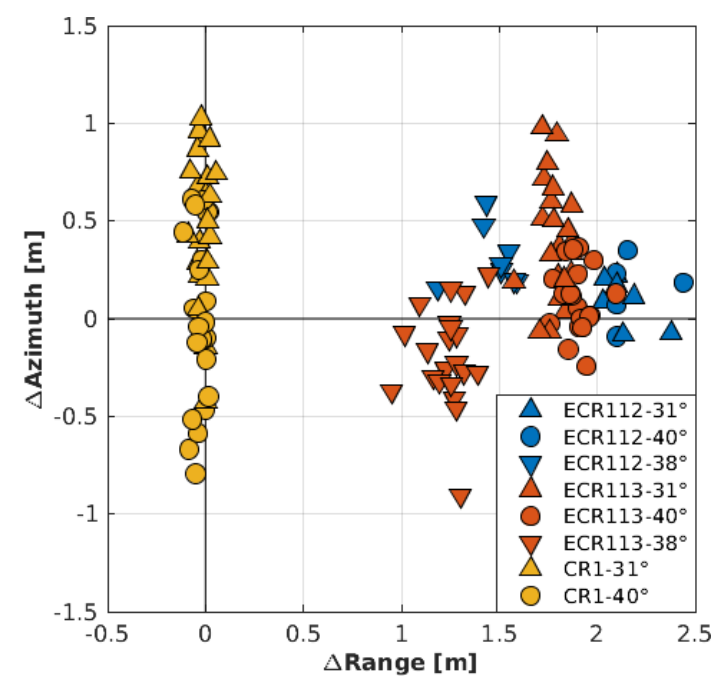

Fig. 3: 2D geolocation results with Sentinel-1 IW SLC data for the two ECRs and CR-las summarized in Table 1.

Because these ECR offsets also differ for the ascending and descending stacks, which are controlled by independent signal chains inside the devices, we attribute them to internal delays caused by electronics. Consequently, the total spread in the range geolocation of the ERCs becomes much larger when analyzed across all available data, see Table 3. Further investigation and proper modelling of this behavior is needed in order to improve the geolocation with the ECRs.

\section{ABSOLUTE 3D LOCALIZATION}

The absolute 3D positions of the installed ECRs and CR-1 can be retrieved from SAR by means of least squares adjustment with additional parameters, based on the stereo SAR concept [2]. For the two ECRs, the localization is carried out in a first step using acquisitions from ascending geometries only, and in a second step using all acquisitions from ascending and descending passes (see section 6). Note that the range and azimuth offsets of Table 1 averaged for both ECRs are used as a preliminary ECR delay characterization to improve the positioning results. The combination of observations from three aspect angles $\left(31^{\circ}\right.$, $40^{\circ}$ and $38^{\circ}$ ) clearly benefits the ECR parameter estimation with respect to the CR's, for which only the ascending passes $\left(31^{\circ}, 40^{\circ}\right)$ can be used. The estimated coordinates are compared against the known reference coordinates and expressed in the local north, east, height frame.

The usage of all geometries significantly improves the ECR positioning results, with the estimated precision of the combined solution being much smaller in comparison to the ascending-only ECR and CR-1 solutions, see Table 2. This is in accordance with the applied method, which is driven by the overall stereo baseline [2]. Because of the high ranging accuracy, the CR-1 performs better when compared to the 
ascending only ECR solutions, which show much larger differences in the east and height components. These differences are due to our preliminary modelling of the ECR delays, but these first results are nevertheless promising. If these delays can be properly characterized by calibration parameters, the ECRs will allow absolute 3D localization with Sentinel-1 with centimeter accuracy.

Table 2: Positioning results of the two ECRs and CR-1. $\Delta N, \Delta E$ and $\triangle H$ are the differences $w . r . t$. the reference coordinates. $\sigma N, \sigma E$, and $\sigma H$ are the estimated precision (95\% confidence) of the least squares solution. Units: $m$.

\begin{tabular}{lcccccc}
\hline \multicolumn{1}{c}{ Target } & $\boldsymbol{\Delta} \mathbf{N}$ & $\boldsymbol{\Delta} \mathbf{E}$ & $\boldsymbol{\Delta} \mathbf{H}$ & $\boldsymbol{\sigma}_{\mathbf{N}}$ & $\boldsymbol{\sigma}_{\mathbf{E}}$ & $\boldsymbol{\sigma}_{\mathbf{H}}$ \\
\hline ECR112 \\
ECR $112_{a}$ & 0.073 & 0.455 & -0.164 & 0.103 & 0.041 & 0.038 \\
ECR113 $_{a, d}$ & 0.153 & 0.324 & -0.273 & 0.137 & 0.273 & 0.237 \\
ECR113 $_{a}$ & 0.146 & 0.560 & 0.204 & 0.113 & 0.240 & 0.174 \\
$\mathrm{CR}-1_{a}$ & 0.174 & -0.041 & 0.019 & 0.196 & 0.132 & 0.098 \\
\hline
\end{tabular}

$a, d$ indicates solution from ascending and descending passes, while $a$ from ascending passes only.

\section{PHASE STABILITY}

To assess the interferometric phase stability we analyzed the spatio-temporal phase differences in the $31^{\circ}$ ascending stack between ECR113 and CR1, using our interferometric wide area processor IWAP [6]. The phase is determined after bandpass oversampling and polynomial fitting of the peak. For comparison, we also analyzed the phase differences between CR-1 and CR-5. Since the distances between the reflectors are all less than $900 \mathrm{~m}$, atmospheric phase screens and other common errors should cancel out.

Fig. 4 shows the phase stability of ECR113, which is quite satisfactory. After removing linear temporal trends (-9.2 $\mathrm{mm} / \mathrm{y}$ for CR5-CR1) presumably caused by the loose grounding of the CRs on grassland, the phase standard deviations are $15.7^{\circ}(0.27 \mathrm{rad})$ for ECR113-CR1 and $10.3^{\circ}$ (0.18rad) for CR5-CR1, corresponding to $1.2 \mathrm{~mm}$ and 0.80 $\mathrm{mm}$. Note that the ECR's phase noise seems to be higher irrespective of its $5.4 \mathrm{~dB}$ higher backscatter value. Due to the high Doppler frequencies $(-1 \mathrm{kHz})$, the phase measurements are highly sensitive to azimuth positioning errors and may also be contaminated from strong signals of nearby buildings. Our results seem to be comparable to the values $0.6 \mathrm{~mm}$ to $1.4 \mathrm{~mm}$ published in [7]. They should be considered as preliminary upper bounds at this stage. Obviously, the phase stability of the ECRs over longer time intervals still needs to be assessed.

\section{SUMMARY}

The use of ECRs has great potential to deliver strong radar markers for geometric positioning and monitoring in environments where conventional CRs cannot be installed. Their power supply and monitoring of presumably aging over the years certainly requires some additional effort.

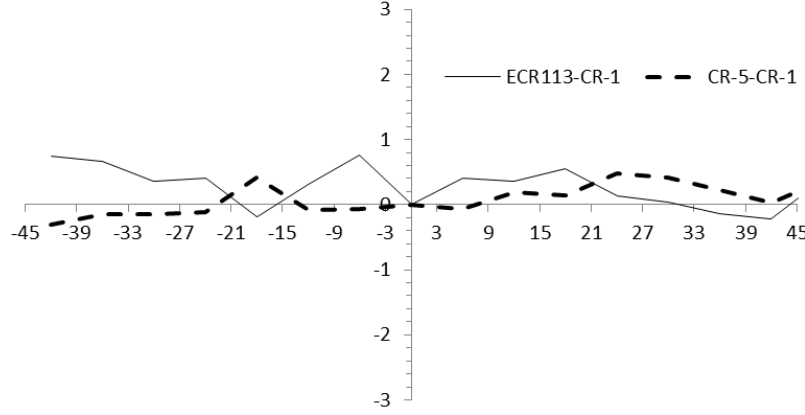

Fig. 4: Phase stability of ECR113 with respect to CRI over 16 cycles (90 days). For comparison, the phase difference between CR5 and CR1 is also shown. (Units: rad, Master: 16.03.2020).

We set up an experiment to perform well controlled longterm measurements and qualify ECRs for operational use. While the ECR phase stability is in good shape, the internal delay behavior degrading the absolute ranging accuracy requires further investigation. If these delay effects can be resolved, the ECRs would also become valuable devices for absolute positioning and geometric characterization of $\mathrm{C}$ Band SAR satellites.

\section{REFERENCES}

[1] M. Eineder, C. Minet, P. Steigenberger, X. Cong, and T. Fritz, "Imaging Geodesy - Toward Centimeter-Level Ranging Accuracy with TerraSAR-X," IEEE Trans. Geosc. Remote Sens., vol. 49, pp. 661-671, 2011.

[2] C. Gisinger, U. Balss, R. Pail, X. Zhu, S. Montazeri, S. Gernhardt, and M. Eineder, "Precise Three-Dimensional Stereo Localization of Corner Reflectors and Persistent Scatterers With TerraSAR-X," IEEE Trans. Geosc. Remote Sens., vol. 53, no. 4, pp. 1782-1802, 2015.

[3] C. Gisinger, U. Balss, H. Breit, A. Schuber, M. Garthwaite, D. Small, T. Gruber, M. Eineder, T. Fritz, N. Miranda, „Recent Findings on the Sentinel-1 Geolocation Accuracy using the Australian Corner Reflector Array“, Proc. IGARSS 2018, pp. 6356-6359, 2018.

[4] A. Schubert, D. Small, C. Gisinger, U. Balss, M. Eineder, "Corner Reflector Deployment for SAR Geometric Calibration and Performance Assessment", CEOS Cal/Val Technical Notes on Calibration Methods Guidelines, issue 1.04, 2017. Online: $\mathrm{http} / / / c a l v a l p o r t a l . c e o s . o r g / c a l i b r a t i o n-m e t h o d s-g u i d e l i n e s$

[5] U. Balss, C. Gisinger, and M. Eineder, "Measurements on the Absolute 2-D and 3-D Localization Accuracy of TerraSAR-X," Remote Sens., vol. 10, no. 656, pp. 1-21, 2018

[6] N. Adam, W. Liebhart, A. Parizzi, F. Rodriguez-Gonzalez, R. Brcic, "Persistent Scatterer Interferometry - Wide Area Product Methodology and Final Characteristics", Terrafirma Stage 3, DLR Remote Sensing Technology Institute, 2012.

[7] P. Mahapatra, H. van der Marel, F.J. van Leijen, S. SamieiEsfahany, R. Klees, R. F. Hanssen, "Insar datum connection using GNSS-augmented radar transponders", Proc. IGARSS 2016, pp. 6867-6870, 2016. 